\title{
Patient Radiation Doses during Diagnostic Cerebral Angiography Procedures: Experience at a Tertiary Level Center of India
}

\author{
Sandeep K Bhatt ${ }^{1}$, Vivek Gupta ${ }^{2}$, Vikas Bhatia ${ }^{3}$, N Khandelwal ${ }^{4}$, SC Bansal ${ }^{5}$, Bhupender S Rana ${ }^{6}$
}

\begin{abstract}
Objective: To assess patient radiation doses during diagnostic cerebral angiography from a tertiary neuro-intervention center. Materials and methods: We studied 161 patients including 149 adults ( 83 male; 66 female) and 12 pediatric patients who underwent diagnostic cerebral angiography (DCA) for suspected or proven intracranial aneurysms which were performed with a biplane unit. Fluoroscopic time, dosearea product (DAP), and total angiographic image frames were obtained and analyzed in males, females and pediatric population separately. Results: Mean fluoroscopic time, the total mean DAP, and total image frames were 11.6 minutes, $105.6 \pm 60 \mathrm{~Gy}-\mathrm{cm}^{2}$, and $295 \pm 82$ frames for diagnostic procedures.

Separate assessment in males, females and pediatric population was made. No significant difference was found between male and female population. Pediatric population, however, showed reduced DAP values (104 vs. $84 \mathrm{Gycm}^{2}$ ) and a number of frames (308 vs. 271.5$)$ with increased fluoroscopic time (11.6 vs. $14.28 \mathrm{~min}$ ) when compared to the adult population.

Conclusion: Radiation dose as measured by DAP, fluoroscopy time and image frames is consistent with previous studies. Further data from other institutes can help in establishing a national registry for radiation exposure and help in framing guidelines for radiation protection.

Keywords: Dose-area product, DSA, Radiation dose.

Journal of Postgraduate Medicine, Education and Research (2019): 10.5005/jp-journals-10028-1315
\end{abstract}

\section{INTRODUCTION}

nterventional neuroradiology has emerged as an increasingly used modality for the treatment of various neurovascular disease processes. These procedures are prolonged and complex and require radiation exposure to staff as well as patients. There is increased awareness and questions about radiation safety for the patient and the staff involved in these diagnostic and therapeutic procedures. $^{1}$

Radiation effects are either deterministic or stochastic. In deterministic effects such as dermatitis and epilation, there is a threshold, and the severity of the effects is a function of the dose. In stochastic effects such as cancer induction and genetic hazards, only the probability of occurrence of the effect is a function of the dose. $^{2}$

Stochastic risks to the patients are usually estimated through the calculation of effective doses from DAP or skin dose. ${ }^{1}$

Aim of the present study was to evaluate the DAP cumulative dose delivered to the patients during diagnostic cerebral angiography procedures.

\section{Materials AND METHOdS}

This prospective observational study was carried out in the Department of Radiology, Postgraduate Institute of Medical Education and Research (PGIMER), Chandigarh, India over 1 year after taking ethical committee approval. Inclusion criteria were patients undergoing diagnostic subtraction angiography for suspected aneurysms where DAP data was available. Exclusion criteria were an incomplete diagnostic procedure or where DAP values could not be ascertained due to technical reasons. There were a total of 161 patients including 149 adults ( 83 male; 66 female) and 12 pediatric patients who underwent DCA for suspected or proven intracranial aneurysm. All examinations were performed on Philips Integris System: Allura Xper FD 20/10 Biplane flat panel

\author{
${ }^{1}$ Senior Technician, ${ }^{2,4}$ Professor, ${ }^{3}$ Assistant Professor, \\ ${ }^{5}$ Assistant Professor, ${ }^{6}$ Physicist \\ ${ }^{1-6}$ Department of Radiodiagnosis and Imaging, Postgraduate institute \\ of Medical Education and Research, Chandigarh India
}

Corresponding Author: Vikas Bhatia, Assistant Professor, Department of Radiodiagnosis and Imaging, Postgraduate Institute of Medical Education and Research, Chandigarh, India, e-mail: drvikasbhatia@ gmail.com

How to cite this article: Bhatt SK, Gupta V, Bhatia V, Khandelwal N, Bansal SC, Rana BS. Patient Radiation Doses during Diagnostic Cerebral Angiography Procedures: Experience at a Tertiary Level Center of India. J Postgrad Med Edu Res 2019;53(2):69-71.

Source of support: Nil

Conflict of interest: None

digital subtraction angiographic system. It has a rotating anode grided maximum rotalix ceramic (MRC) X-ray tube, with anode material zirconium, $\mathrm{Rn}$, tungsten; diameter of anode disk is 200 $\mathrm{mm}$ with anode rotation speed $4200 \mathrm{rpm}$. Anode angle 9-110 and two focal spots. Exposure factors used were KV-80, mAs-29 for adults and kv-80,mAs- 8 for pediatric patients. These were adjusted at the time of acquisition for the variable thickness of the patient. Two fluoroscopic modes were used $30 \mathrm{fr} / \mathrm{s}$ (used in pediatric procedures at low KV and mA settings) and 15/fr/s used for adults to reduce radiation dose to patients. DAP was measured using the new KermaX ${ }^{\circledR}$ plus TinO-Two in one-multifunctional measuring system which combines a real-time dosimeter and a DAP meter. It is a large flat plate ionization chamber fixed to the collimator on the $x$-ray tube and through which the whole X-ray beam passes.

\section{Results}

Table 1 shows minimum, maximum and average number of runs taken, images per examination, fluoroscopy time and DAP per examination.

() The Author(s). 2019 Open Access This article is distributed under the terms of the Creative Commons Attribution 4.0 International License (https://creativecommons. org/licenses/by-nc/4.0/), which permits unrestricted use, distribution, and non-commercial reproduction in any medium, provided you give appropriate credit to the original author(s) and the source, provide a link to the Creative Commons license, and indicate if changes were made. The Creative Commons Public Domain Dedication waiver (http://creativecommons.org/publicdomain/zero/1.0/) applies to the data made available in this article, unless otherwise stated. 
Table 1: Minimum, maximum and average number of runs taken, images per examination, fluoroscopy time and DAP per examination

\begin{tabular}{llll} 
Category & Minimum & Maximum & Mean \\
\hline Runs taken & 5 & 21 & 9.43 \\
Images/exam & 40 & 1728 & 295 \\
Fluoro time & $2.25 \mathrm{~min}$ & $55.59 \mathrm{~min}$ & $11.6 \mathrm{~min}$ \\
DAP $\left(\mathrm{Gy}-\mathrm{cm}^{2)}\right.$ & 16.143 & 541.183 & 105.096 \\
\hline
\end{tabular}

Table 2: Variation of number of runs, images per exam, fluoroscopy time, DAP/exam, DAP/image in male, female and pediatrics patients during the procedure

\begin{tabular}{|c|c|c|c|}
\hline Average & Male & Females & Children \\
\hline Runs taken & 9.6 & 9.48 & 8.75 \\
\hline Images/exam & 328.67 & 289.98 & 271.5 \\
\hline Fluoro time (min) & 11.42 & 11.78 & 14.28 \\
\hline DAP/exam Gycm² & 111.58 & 97.44 & 84.32 \\
\hline DAP/image $\mathrm{mGycm}{ }^{2}$ & 339.49 & 336.027 & 310.59 \\
\hline
\end{tabular}

Table 2 shows variation of number of runs, images per exam, fluoroscopy time, DAP/exam, DAP/image in male, female and pediatrics patients during the procedure.

Graph 1 shows DAP $\left(\mathrm{mGycm}^{2}\right)$ among male, female and children per examination.

Our data is consistent with previous published reports ${ }^{3-7}$ which have estimated DAP, fluoroscopy time and number of frames in patients undergoing diagnostic cerebral angiography as shown in Table 3.

\section{Review}

Intervention neuroradiology procedures for both diagnostic and therapeutic indications have increased recently and are potentially associated with considerable radiation exposure which can cause both stochastic and deterministic effects. Thus it is of paramount importance to characterize risks to patients undergoing these procedures especially in case of pediatric population. ${ }^{8}$

Radiation dose with such procedures is difficult to due to the variability of institutional protocols, procedure-related complexities, differences in equipment and experience of the interventional neuroradiologist. The aim of this study was to calculate radiation exposure through DAP measurements in patients undergoing diagnostic cerebral procedures to provide reference data in Indian population at an apex tertiary institute and also to compare it with previously reported data.

A major cause of standardization of procedures and dose measurements is lack of standardization among hospitals, different

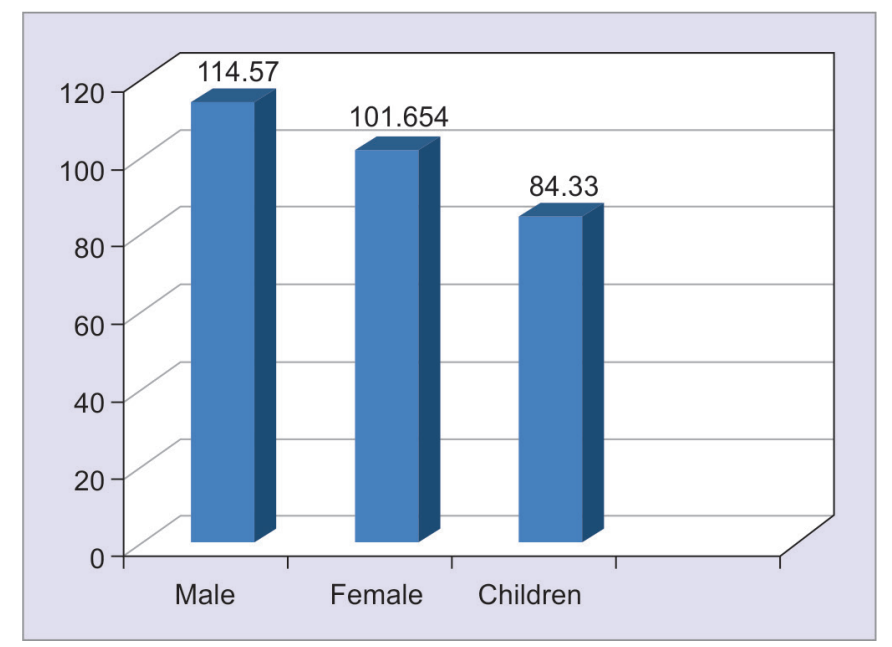

Graph 1: DAP $\left(\mathrm{mGycm}^{2}\right)$ among male, female and children per examination

technique, and experience of interventionists and variability in machines which use different methods for dosage measurement. Various measurement indices which are commonly used are DAP, fluoroscopy time, ED, entrance skin dose and kerma. ${ }^{1}$

Dose-area product can be a useful tool to determine the radiation exposure during neurointervention procedures which can be used to assess for possible radiation injuries on follow-up. The DAP meter uses an ionization chamber which is fixed to the collimator on the X-ray tube and through which the whole X-ray beam passes. DAP is measured in $\mathrm{Gy}-\mathrm{cm}^{2}$ and is a function of the cross section of an X-ray beam and the X-ray exposure at the level of the collimator. Conversion factors have been previously described which can be used for calculation of effective dose from DAP. ${ }^{9,10}$

DAP cannot accurately delineate individual patient risk, but it may provide estimation about the risk of stochastic and deterministic effects by providing the effective dose and entrance skin dose. ${ }^{3}$

In addition, we have estimated these values by separating our study population into male, female and pediatric population which has not been extensively studied before. No significant difference was found between male and female population. Pediatric population, however, showed reduced DAP values ( $\left.104 \mathrm{vs} .84 \mathrm{Gycm}^{2}\right)$ and a number of frames ( 308 vs. 271.5) with increased fluoroscopic time (11.6 vs. 14.28 $\mathrm{min}$ ) when compared to the adult population. A probable explanation for lower DAP values in the pediatric population is the use of low KV and $m$ As settings in the pediatric population.

The main aim of this study was to provide a reference range in Indian population for radiation exposure as measured at a tertiary level care center with vast experience in neurointervention procedures.

Table 3: Review of previous studies with diagnostic cerebral angiography for intracranial aneurysms

\begin{tabular}{lllll}
\hline & & DAP $\left(\mathrm{Gy}-\mathrm{cm}^{2}\right)$ & Fluoroscopy time $($ Min) & Number of frames \\
\cline { 2 - 5 } Reference & No. of patients & Mean & Mean & Mean \\
\hline Present study & 161 & 105.1 & 11.6 & 295 \\
Chang et al. ${ }^{3}$ & 439 & 136.6 & 12.6 & 251 \\
D'Ercole et al. $^{4}$ & 100 & 142.1 & 9.9 & 220 \\
Aroua et al. $^{5}$ & 91 & 121 & 12.6 & - \\
Verdun et al. $^{6}$ & 91 & 107 & - & - \\
Brambilla et al. $^{7}$ & 188 & 158 & 13.7 & - \\
\hline
\end{tabular}


There are a few drawbacks in our study. Data presented here is experience from a single tertiary-level center with its own set of protocols during such procedures and may vary from other institutes.

These values are for a biplane machine from a single vendor and values may vary from other institutes having different machines. These investigations were carried out by different operators and slight variation due to the skill and expertise of the operator may alter the radiation exposure.

However, we believe that this information can help in future risk estimation and prognostication of radiation exposure and help in the implementation of radiation safety measures. A future reference data from other premier institutes can help in standardization of radiation exposure and risks in patients undergoing such procedures.

\section{References}

1. Geise, RA, O'Dea TJ. Radiation dose in interventional fluoroscopic procedures. Appl. Radiat. Isot 1999:50:173-184.

2. International Commission on Radiological Protection (ICRP). 1990 Recommendations of the International Commission on radiological protection. ICRP Publication 60. Oxford, England: Permagon Press 1991;21:(1-3):54.
3. Chun CW, Kim B, Lee CH, et al. Patient Radiation Dose in Diagnostic and Interventional Procedures for Intracranial Aneurysms: Experience at a Single Center. Korean J Radiol 2014;15(6):844-849.

4. D'Ercole L, Thyrion FZ, Bocchiola M, et al. Proposed local diagnostic reference levels in angiography and interventional neuroradiology and a preliminary analysis according to the complexity of the procedures. Phys Med 2012;28:61-70.

5. Aroua A, Rickli H, Stauffer JC, et al. How to set up and apply reference levels in fluoroscopy at a national level. Eur Radiol 2007;17:1621-1633.

6. Verdun FR, Aroua A, Trueb PR, et al. Diagnostic and interventional radiology: a strategy to introduce reference dose level taking into account the national practice. Radiat Prot Dosimetry 2005;114:188191.

7. Brambilla $M$, Marano G, Dominietto $M$, et al. Patient radiation doses and references levels in interventional radiology. Radiol Med 2004;107:408-418.

8. Bor D, Cekirge S, Turkay T, et al. Patient and staff doses in interventional neuroradiology. Radiat Prot Dosimetry 2005;117:62-68.

9. Nickoloff EL, Lu ZF, Dutta AK, et al. Radiation dose descriptors: BERT, COD, DAP, and other strange creatures. Radiographics 2008;28: 1439-1450.

10. Bor D, Sancak T, Olgar T, et al. Comparison of effective doses obtained from dose-area product and air kerma measurements in interventional radiology. Br J Radiol 2004;77:315-322. 